Revue des patrimoines

Le cheval et ses patrimoines (1ère partie)

\title{
Le patrimoine équestre des écoles nationales vétérinaires françaises
}

\section{Christophe Degueurce}

\section{(2) OpenEdition}

\section{Journals}

Édition électronique

URL : http://journals.openedition.org/insitu/9645

DOI : 10.4000/insitu.9645

ISSN : 1630-7305

\section{Éditeur}

Ministère de la culture

Référence électronique

Christophe Degueurce, «Le patrimoine équestre des écoles nationales vétérinaires françaises », In Situ [En ligne], 18 | 2012, mis en ligne le 01 août 2012, consulté le 23 avril 2019. URL : http:// journals.openedition.org/insitu/9645; DOI : 10.4000/insitu.9645

Ce document a été généré automatiquement le 23 avril 2019

\section{(c) (i) () $\Theta$}

In Situ Revues des patrimoines est mis à disposition selon les termes de la licence Creative Commons Attribution - Pas d'Utilisation Commerciale - Pas de Modification 4.0 International. 


\title{
Le patrimoine équestre des écoles nationales vétérinaires françaises
}

\author{
Christophe Degueurce
}

1 La formation vétérinaire est dispensée en France dans quatre écoles, pour certaines très anciennes et qui ont pu souvent conserver un patrimoine pédagogique, scientifique et artistique fortement marqué par le cheval ${ }^{1}$. Il est intéressant d'évoquer la création de ces écoles et leurs déplacements éventuels car ils ont conditionné en grande partie la conservation du patrimoine. La plus ancienne est celle de Lyon, la toute première école vétérinaire au monde, créée en 1761 par Claude Bourgelat, écuyer du roi tenant l'académie d'équitation de cette ville. D'abord logée dans une ancienne auberge du faubourg de La Guillotière, cette école a déménagé en 1796 pour rejoindre les quais de la Saône à Vaise. Magnifiquement logée dans l'ancien couvent des Deux-Amants, cette école fut à nouveau déménagée en 1978 pour s'excentrer à l'Ouest de Lyon, dans des locaux plus adaptés aux besoins de la formation et des cliniques. La seconde fut celle de Paris dont l'établissement fut décidé en 1764 et se concrétisa par le départ pour la capitale de deux enseignants et de quelques élèves de Lyon en juillet 1765. Ils commencèrent à préparer des pièces anatomiques dans une rue proche de la porte Saint-Martin avant de rejoindre, en 1766, le site du château d'Alfort, près de Charenton, tout juste préparé à recevoir des élèves par Jacques-Germain Soufflot. L'école actuelle occupe toujours la parcelle d'origine. La troisième date de 1825 et fut établie à Toulouse, dans le quartier de Matabiau. Elle occupait un imposant bâtiment en briques, avec des cours, un jardin botanique... Cette école quitta en 1964 le centre ville pour la périphérie, à proximité de l'aéroport de Blagnac. Enfin, l'École vétérinaire de Nantes fut créée à la fin des années 1970, dans des locaux modernes très adaptés.

2 Les trois premières écoles ont été marquées par le cheval, un animal indissociable des débuts de la médecine vétérinaire. À notre connaissance, la première personne a avoir eu le titre de "vétérinaire » fut Jean Héroard, médecin de formation, qui fut «médecin en l'art vétérinaire » du roi Charles IX. Et le créateur des deux premières écoles, Claude Bourgelat, était écuyer et versé dans l'hippiatrie, c'est-à-dire la médecine et la chirurgie du cheval, un besoin naturel pour ces praticiens de l'art équestre qui, dressant souvent pendant 
plusieurs années leurs chevaux, ne pouvaient accepter qu'ils soient amoindris par des maladies ; ils furent, à côté des traditionnels maréchaux, les promoteurs d'une hippiatrie modernisée, fixée par l'écrit, fondée sur des raisonnements s'appuyant notamment sur ceux de la médecine de l'Homme. Ceci explique que l'enseignement des écoles du XVIII siècle ait été essentiellement consacré à l'animal noble, l'animal connu, le cheval, et ceci même si le roi de France avait clairement assigné aux deux premières écoles la mission de soigner en premier lieu les bestiaux.

3 L'objet de cette présentation n'était pas de présenter un inventaire exhaustif du patrimoine mais de dégager quelques tendances pour envisager de façon prospective ce qui pourrait être mis en œuvre pour en améliorer la sauvegarde et en accroitre la visibilité.

\section{La méthode}

4 Concrètement, les trois directeurs des écoles autres qu'Alfort (notée ENVA) ont été contactés et ont souvent relayé ma demande d'information concernant le patrimoine des établissements dont ils ont la charge. À Lyon, le professeur J.-L. Cadoré de VetAgroSup (ex École nationale vétérinaire de Lyon - notée $E N V L$ ) m'a fourni une liste des objets d'intérêt patrimonial de cette école; l'ensemble occupe sept feuillets et montre des objets scientifiques et techniques, des pièces anatomiques, des souvenirs tels que des bustes ou des médaillons, ces objets étant dispersés dans les différents services de l'établissement. En revanche, l'inventaire des collections pédagogiques est parcellaire; la collection Lesbre-Tagand de tératologie ou encore les pièces d'anatomie comparée, certains ensembles, comme les fers, ne sont pas détaillés.

5 La professeure Dominique Fanuel d'Oniris (ex École nationale vétérinaire de Nantes notée $E N V N)$ a marqué son vif intérêt pour la démarche et m'a indiqué qu'ils disposent d'un cheval clastique d'Auzoux, déposé au Muséum d'histoire naturelle de Nantes.

6 Ma tâche à l'École nationale vétérinaire de Toulouse (notée ENVT) a été grandement facilitée par l'impressionnant travail réalisé par deux étudiants ayant réalisé leurs thèses sur la sauvegarde du patrimoine de cet établissement. Anne-Laure Sivignon a réalisé son travail de thèse sur l'inventaire des objets scientifiques et techniques, mettant en place une procédure d'inventaire et sauvegardant une partie de ce patrimoine dans une salle de conservation ${ }^{2}$. Vincent Blanchard <lien vers http://oatao.univ-toulouse.fr/4692> a quant à lui effectué le même travail sur les "objets d'art", ce vocable réunissant dans son mémoire les œuvres d'art à part entière et les collections pédagogiques, souvent faites de moulages, de squelettes, etc. ${ }^{3}$

7 Quant à l'École nationale vétérinaire d'Alfort, les choses étaient assez simples dans la mesure où, le musée Fragonard étant Musée de France au sens de la Loi de 2002, il dispose d'un inventaire informatisé qui rassemble l'essentiel du patrimoine connu.

\section{Quelques résultats}

\section{Les collections pédagogiques liées au cheval}

8 À l'ENVA, le musée Fragonard développe ses collections sur $500 \mathrm{~m}^{2}$, les objets étant mis en valeur dans de grandes vitrines créées pour le musée lors de son réaménagement en 1902. 
Ce sont aujourd'hui 4167 pièces qui sont présentées au public, dont 1651 sont consacrées au cheval, soit environ $40 \%$ de la collection présentée. Dans le détail, 356 spécimens anatomiques dévoilent la structure du cheval, certains étant connus dans le monde entier comme le cavalier (vers 1765) (fig. $\left.\mathbf{n}^{\circ} \mathbf{1}\right)$ d'Honoré Fragonard (1732-1799) présentant un jeune garçon disséqué juché sur un cheval pareillement dépouillé. Cette pièce permettait notamment, à une époque où l'anatomie comparée était en plein développement, de montrer les analogies entre deux espèces pourtant considérées comme très dissemblables. À côté des pièces d'anatomie naturelle, plusieurs centaines de moulages en cire et plus souvent en plâtre dévoilent dans leurs chauds coloris les détails de l'anatomie de chaque organe. En sus, les collections recèlent un grand nombre de dents et mâchoires utilisées pour la pathologie comme pour l'usure dentaire.

Figure 1

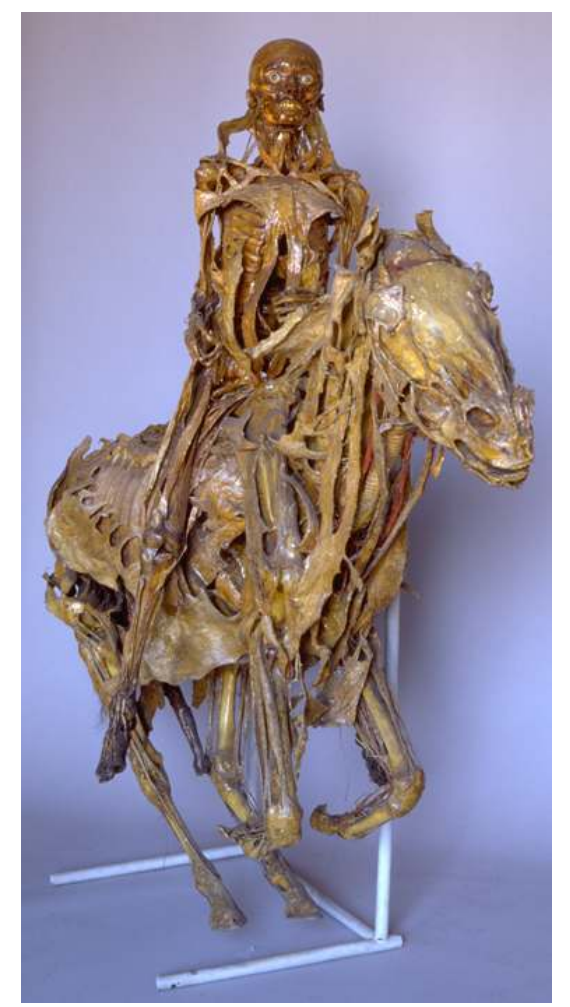

Le cavalier, par Honoré Fragonard (entre 1765 et 1771). Collections du Musée Fragonard.

(c) Christophe Degueurce.

9 À côté de l'anatomie normale, 693 lésions équines révèlent indirectement ce que pouvaient être les conditions de vie des équidés aux XVIII ${ }^{\mathrm{e}}$ et XIX ${ }^{\mathrm{e}}$ siècles, quand le cheval était le moteur de l'économie, soumis à un travail long et inducteur de lésions chroniques que l'on peine à imaginer aujourd'hui. Elles reflètent indirectement la valeur financière de cet animal qui était mené jusqu'à ses capacités ultimes. Certaines lésions ont d'ailleurs tout bonnement disparu du répertoire pathologique contemporain, à l'image des maladies professionnelles comme l'ostéofibrose nutritionnelle du cheval de meunier ${ }^{4}$, ou encore les phénomènes de fusions vertébrales si courantes chez les chevaux de traction (fig. $\left.\mathbf{n}^{\circ} 2\right)$. D'autres objets évoquent les conditions de maintien des animaux, à l'image des 190 calculs digestifs, salivaires, rénaux, vésicaux... de chevaux, hérités des XVIII ${ }^{\mathrm{e}}$ et XIX ${ }^{\mathrm{e}}$ 
siècles, probablement liés à l'insuffisance en eau de boisson, dont souffraient ces animaux. Enfin, 153 flacons contiennent des parasites du cheval.

Figure 2

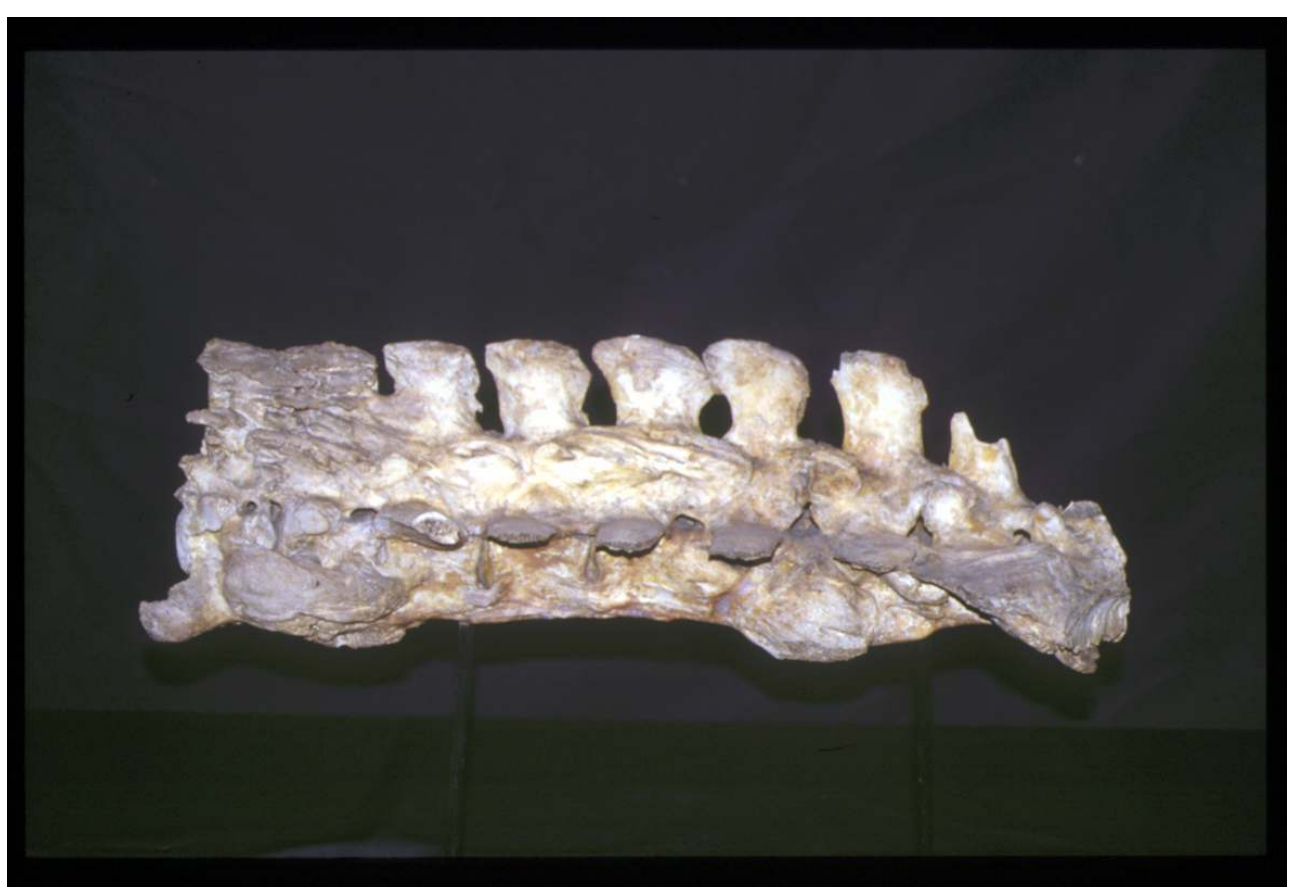

Ankylose de la colonne vertébrale. Collections du Musée Fragonard.

(C) C. Degueurce.

L'ENVL dispose de collections d'anatomie et de tératologie ${ }^{5}$ qui ne sont, à ma connaissance, pas inventoriées ou dont l'inventaire n'est pas publié. Ces collections sont d'une importance majeure car elles ont servi de point d'appui au fameux Traité de tératologie publié par Lesbre ${ }^{6}$. L'ENVT a en revanche un musée anatomique servant aux élèves et contenant, par exemple, 23 moulages en carton peint, très originaux et d'une grande beauté, qui décoraient originellement la salle de dissection de l'ancienne école, à côté de squelettes et autres modèles anatomiques. Également, l'ENVT dispose d'une collection d'environ 160 fers et une importante collection de vues photographiques portant sur diverses pathologies.

Les quatre écoles vétérinaires ont en commun de disposer chacune d'un cheval clastique d'Auzoux (fig. $\mathbf{n}^{\circ} \mathbf{3}$ ). Ces mannequins, datés de la seconde moitié du XIX siècle, étaient constitués de plusieurs centaines de pièces faites de papier mâché renforcé par des armatures métalliques, et richement colorées. Entièrement démontables, ils révélaient les plus petites formations anatomiques et permettaient de mener une dissection sans recours au cadavre. Ceci était un élément important à une époque où l'absence de produits anti-infectieux faisait courir un risque mortel au dissecteur se blessant avec un scalpel souillé. Il en existait de deux sortes : le cheval complet, dans lequel chaque muscle pouvait être isolé, et le cheval incomplet, bien moins complexe et centré sur les viscères. Ces chevaux sont très rares car très fragiles, et l'ENVL peut s'enorgueillir de posséder peut-être le plus bel exemplaire connu du modèle complet. Celui de l'ENVT est un peu endommagé mais tout de même en très bon état. Quant à celui de l'ENVA, modèle 
incomplet cette fois, il était très endommagé et a été restauré au cours du printemps 2012. Celui de l'ENVN est déposé au muséum de la ville.

Figure 3

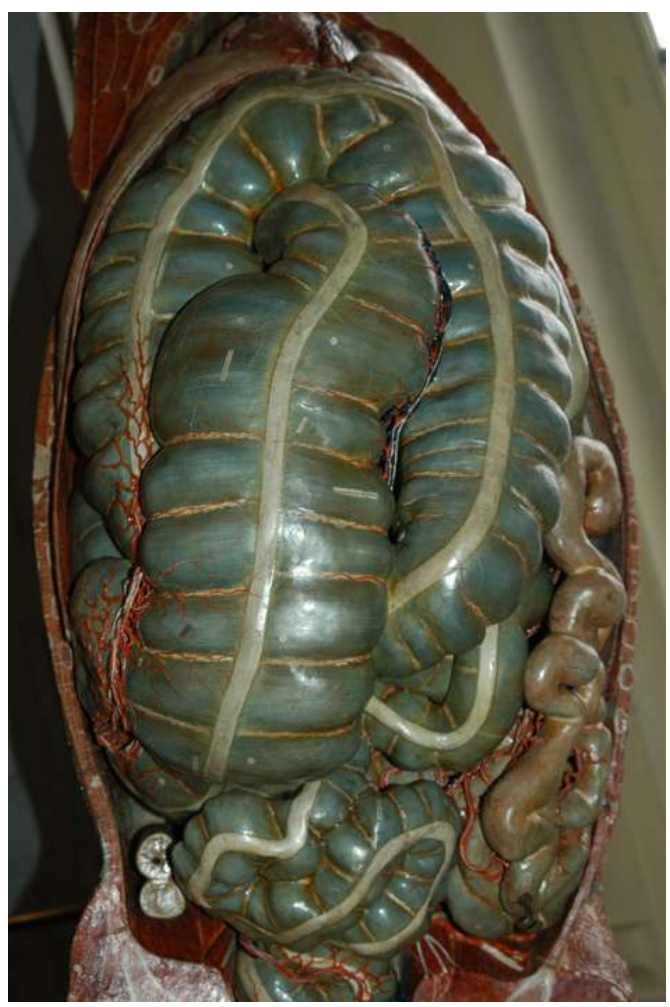

Vue des organes abdominaux du cheval d'Auzoux, après ouverture (vers 1850). Collections du Musée Fragonard.

(c) Christophe Degueurce.

\section{Les collections scientifiques et techniques}

12 L'ENVA est peu dotée avec seulement 92 objets, les collections d'objets scientifiques et techniques ayant été probablement jetées au siècle dernier. Elles se reconstituent aujourd'hui avec les multiples dons que le musée reçoit, mais l'inventaire, en cours dans ce domaine, n'est pas achevé ; le nombre d'objets à inventorier est supérieur à 400 .

L'ENVT a inventorié 467 objets, dont 130 instruments. Quatre-vingt sept pour cent des objets inventoriés ont été placés en salle de sauvegarde et $50 \%$ sont marqués.

L'ENVL possède de nombreux appareillages scientifiques notamment liés au professeur Chauveau (1827-1917), probablement un des scientifiques les plus éminents que les écoles vétérinaires aient produit. Ce court article n'est pas le lieu de présenter ses travaux mais son nom reste attaché à de très nombreuses études portant sur la physiologie, l'immunologie, la cardiologie... On lui doit notamment une collaboration avec ÉtienneJules Marey et l'usage de la méthode graphique pour documenter les pressions dans les cavités du cœur du cheval (fig. $\mathbf{n}^{\circ} \mathbf{4}$ ). Il ne faut pas oublier qu'à une époque où la miniaturisation n'avait pas débuté, cet animal volumineux et assez placide qu'est le cheval constituait un modèle expérimental de choix. L'ENVL conserve de nombreux instruments, tracés, et même une maquette du laboratoire de Chauveau. 
Figure 4

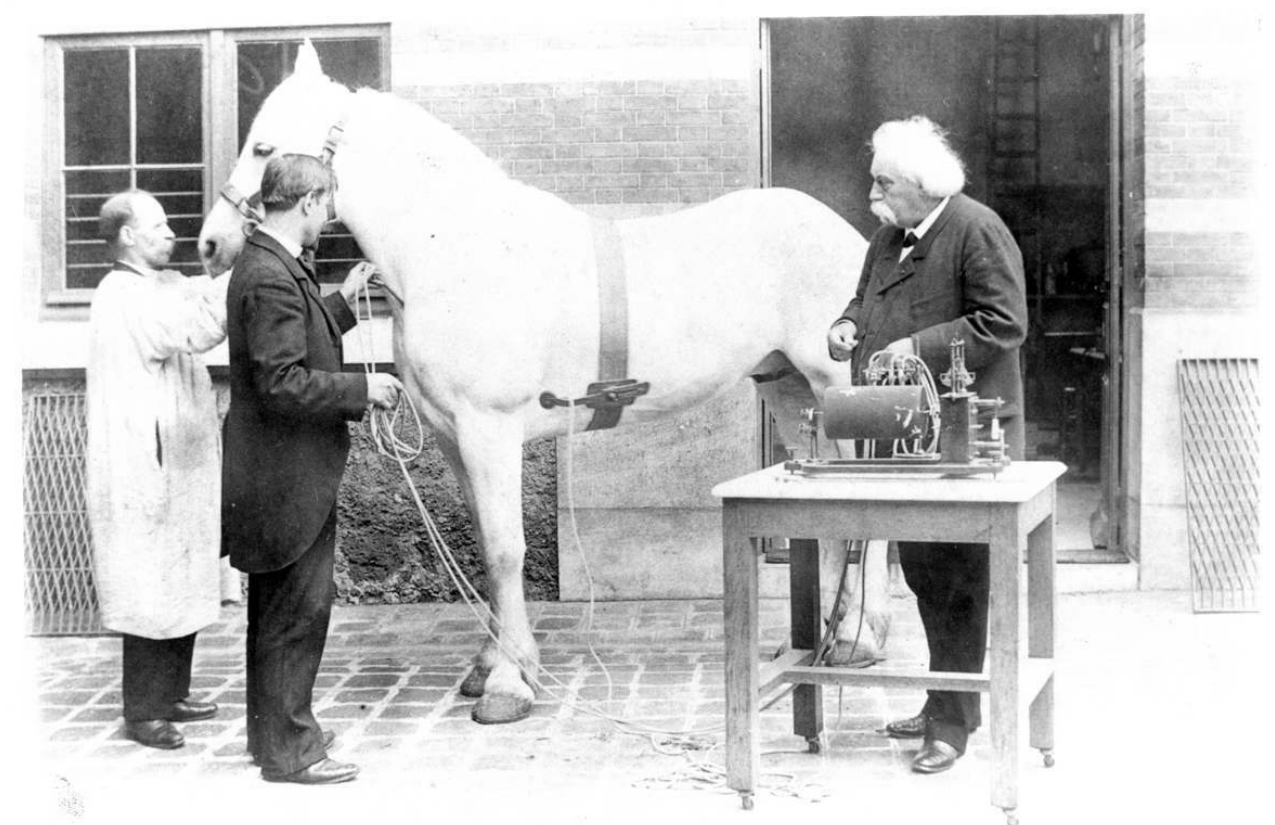

Auguste Chauveau mesurant les pressions dans les ventricules du cœur d'un cheval (vers 1890).

(C) UP Physiologie ENVA.

\section{Les ressources documentaires} référence et, chose appréciable, leurs fonds historiques sont complémentaires. L'ENVA et l'ENVL se sont associées à la Bibliothèque Inter-Universitaire de Santé (ex BIUM) pour présenter sur son site un corpus de textes de référence en médecine vétérinaire. Avec 240 ouvrages offerts à la consultation, il constitue un des plus importants ensembles de la collection Medic@<lien vers http://www2.biusante.parisdescartes.fr/livanc/? intro=alfort\&statut=charge>. L'ENVT a, quant à elle, mis en ligne quelques 210 documents numérisés sur Wikimedia Commons dont 95 thèses anciennes et une très belle iconographie.

16 La situation des archives est en revanche mal connue. Celles de l'ENVA ont été déposées aux archives départementales du Val-de-Marne (jusqu'en 1979) et sont librement consultables dans cette institution.

\section{Les objets d'art mettant en scène des chevaux}

17 L'ENVA dispose d'un beau patrimoine, en grande partie inscrit à l'inventaire des monuments historiques. C'est ainsi que l'on peut y trouver le travail de Bourgelat, cage de contention commandée en 1770 pour sa nouvelle école d'Alfort, de nombreux tableaux tels, qu'entre autres, La leçon d'anatomie chez Lafosse, par Sollier, vers 1770, où l'on voit de hauts personnages suivre une démonstration d'anatomie portant sur le pied du cheval, des sculptures comme le buste de l'étalon La Truffe (fig. $\mathbf{n}^{\circ} 5$ ), cheval du duc d'Angoulême, réalisé en 1826 par Jacques-Nicolas Brunot, ou encore le plâtre de concours d'un 
audacieux monument aux morts mettant en scène deux centaures symbolisant le français et l'allemand, sous les traits de Vercingétorix et de César, monument érigé en face de l'École de Cavalerie de Saumur.

Figure 5

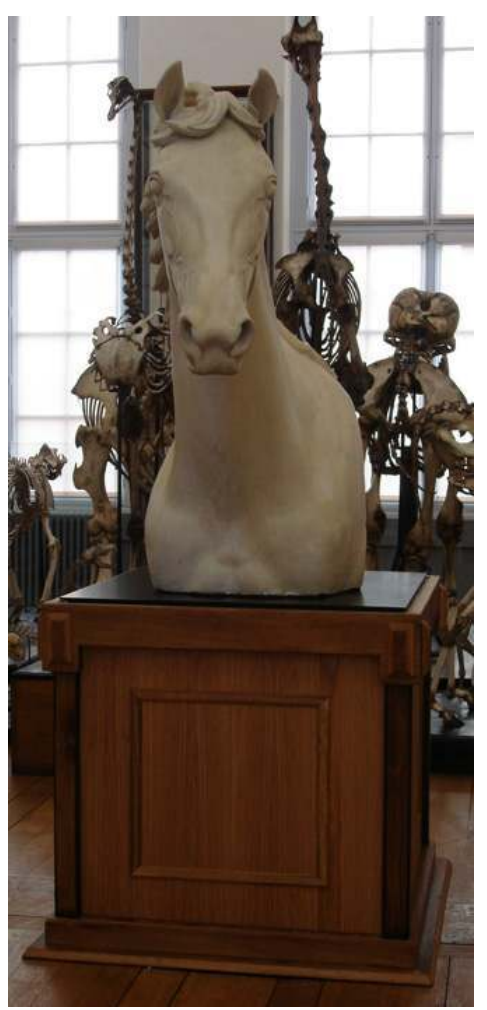

Buste en plâtre de l'étalon La Truffe, par Jacques-Nicolas Brunot. Collections du Musée Fragonard. (c) Christophe Degueurce.

18 Si l'ENVL n'a conservé que quelques éléments du patrimoine artistique de son ancienne école, l'ENVT dispose en revanche d'un chef d'œuvre du XIXe siècle, Le condamné de Montfaucon d'Emmanuel Frémiet. Cette œuvre poignante montre un cheval encapuchonné, le membre brisé, dans le charnier de Montfaucon, prêt à être abattu (fig. $\mathbf{n}^{\circ}$ 6) ${ }^{7}<$ lien vers http://www.revmedvet.com/2003/RMV154_139_152.pdf> 


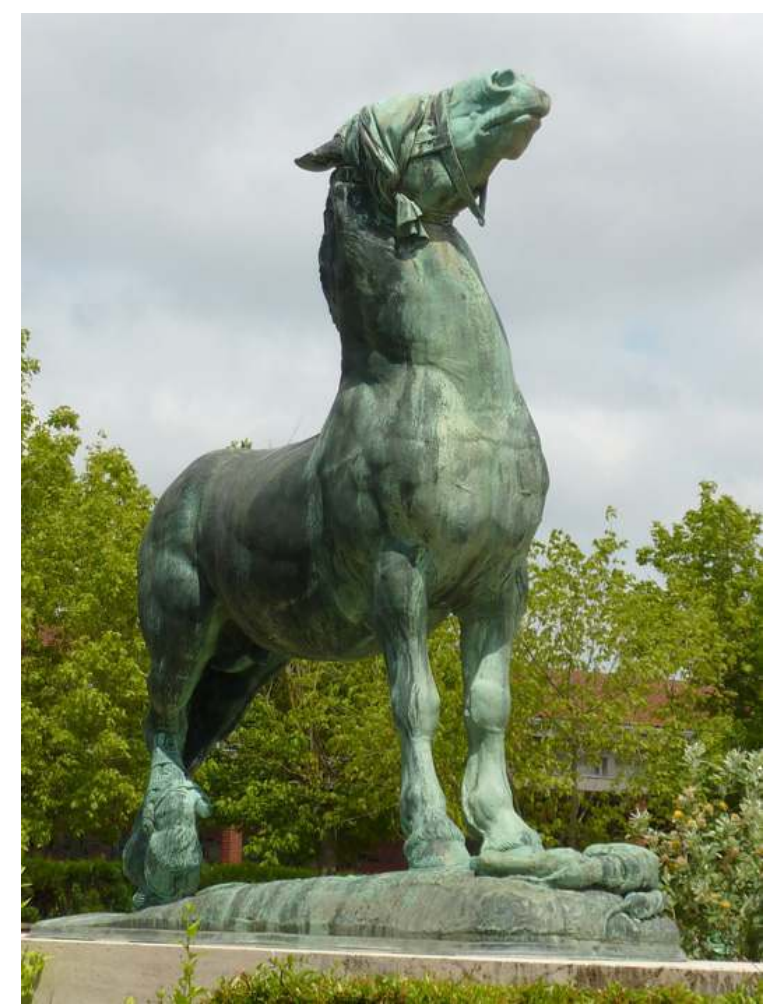

Le condamné de Montfaucon, par Emmanuel Frémiet. Collections de l'ENVT.

(C) Sylvie Chastant-Maillard.

\section{Quelques pistes de réflexion}

19 Le premier examen laisse apparaître des pertes probablement importantes sur le plan patrimonial dans les deux écoles ayant connu des déménagements. L'étude du cas particulier du musée Fragonard, pourtant très préservé tout au long de son existence, illustre ce que peut être la dérive du patrimoine dans un établissement pourtant stable et fondant grandement sa renommée sur un passé prestigieux. Le récolement des collections en 2005 dans le but de candidater à l'appellation Musée de France a été l'occasion de mesurer la déperdition des collections. En 1912, l'inventaire mentionnait 8871 objets mais seuls 2823 étaient présents lors du récolement, soit $32 \%$ du total originel. Des ensembles complets étaient manquants comme la minéralogie, l'agriculture et les ustensiles de chirurgie. In fine, ce sont 5130 objets qui figuraient à l'inventaire finalisé en 2006, dont 2307 inscrits pour la première fois. Ce constat émis sur une collection bénéficiant d'un lieu de présentation unique, non dispersée, dans un établissement qui ne fut pas déplacé, laisse imaginer ce qu'il est advenu du patrimoine de l'ENVL et de l'ENVT lors de leurs mouvements successifs.

Face à cette situation, une politique patrimoniale cohérente pourrait être proposée ; il serait logique de mettre en œuvre un inventaire général des collections des quatre écoles, fondé sur des inventaires d'établissements compatibles entre eux et créés selon les standards des Musées de France. Ceci permettrait de proposer une offre patrimoniale globale aux acteurs culturels (concepteurs d'expositions, éditeurs, journalistes, cinéastes...) et de révéler des sites patrimoniaux insoupçonnés. Ceci mènerait 
probablement à une politique culturelle partagée par des entités qui n'ont pas, chacune, la taille critique pour être visibles. Une telle campagne de récolement devrait être l'occasion pour les écoles qui n'y ont pas procédé de bâtir une politique de conservation fondée sur le regroupement des objets dispersés dans des locaux dédiés, à l'image de la salle de sauvegarde de l'ENVT, leur conditionnement et la création d'une responsabilité en matière culturelle. L'ENVN aurait probablement un rôle important à jouer en matière de collecte et de sauvegarde du patrimoine contemporain, une question actuellement délaissée par tous les établissements.

21 Cette question de la gestion du patrimoine est infiniment politique car elle porte une partie importante de l'identité de ces institutions. Pendant plusieurs décennies, le patrimoine des institutions d'enseignement et de recherche a été considéré comme une charge, un fardeau renvoyant une image négative, désuète, dans des cercles où l'objectif est la projection dans le futur. Et pourtant, les artistes et les scientifiques ont tous en commun d'appuyer leurs avancées sur les travaux de leurs prédécesseurs. Paradoxalement, si les professionnels de l'art ont su développer une brillante histoire, multiplier les références au passé et appuyer ce processus sur la conservation des œuvres de leurs prédécesseurs, les scientifiques ont volontiers abandonné ou ignoré les travaux sur lesquels ils se juchent pour aborder les problématiques du futur. Ce faisant, ils ont nié leur identité propre et sabordé la dimension culturelle de leur travail. Mais les temps changent et il n'est que d'observer la dimension sociale des appels à projets des plans Campus ou des IDEX pour comprendre que la société civile attend de ces institutions une ouverture et un retour direct, culturel, sur la population.

Le patrimoine matériel est un élément et un atout important. S'agissant des écoles nationales vétérinaires françaises, il est encore temps de mettre en œuvre ce processus de sauvegarde.

\section{NOTES}

1. - J'adresse mes remerciements à Jean-Luc Cadoré, VetAgroSup; Dominique Fanuel, Oniris ; Anne-Laure Sivignon, École nationale vétérinaire de Toulouse.

2. - SIVIGNON, Anne-Laure. Inventaire du patrimoine culturel de l'École Nationale Vétérinaire de Toulouse: les objets scientifiques et techniques. Thèse de médecine vétérinaire, Toulouse. En fin de rédaction. La thèse n'est pas encore soutenue mais sera consultable sur le site http://oatao.univtoulouse.fr.

3. - BLANCHARD, Vincent. Inventaire du patrimoine culturel de l'École Nationale Vétérinaire de Toulouse : les objets d'art. Thèse de médecine vétérinaire. Toulouse 3, 2011, $159 \mathrm{p}$.

4. - Également dénommée «maladie de la tête d'hippopotame ».

5. - Discipline qui s'attache à l'étude des monstres.

6. - LESBRE, François-Xavier. Traité de tératologie de l'homme et des animaux domestiques. Paris: Vigot frères, 1927, $342 \mathrm{p}$.

7. - BODIN, G. « Le condamné de Montfaucon; Salon de Paris 1852 : message abscons d'Emmanuel Frémiet aux vétérinaires? ». Revue Médecine Vétérinaire, 2003, 154, 2, p. 139-152. 


\section{RÉSUMÉS}

Trois des quatre écoles nationales vétérinaires françaises sont très anciennes, et ont fondé historiquement leur développement sur les soins aux chevaux. Elles ont ainsi accumulé un patrimoine lié au cheval, pédagogique, scientifique, technique, et même artistique tout à fait exceptionnel, dont un premier inventaire est dressé ici. La situation est hétérogène avec une disparité des conditions de conservation, de traitement et de valorisation des collections. Ces patrimoines complémentaires gagneraient à bénéficier d'une gestion commune sur la base des normes des Musées de France.

Three of the four French national veterinary schools are very old and have established historically their development on the care of horses. They have accumulated large collections related to horses, dealing with educational, scientific, technical and even artistic skills. An initial inventory is drawn here. The situation is mixed with a disparity of conservation, treatment and recovery of collections. These additional assets could benefit from common management based on the standards of the Museums of France.

INDEX

Mots-clés : cheval, vétérinaire, collections pédagogiques, collections scientifiques, collections techniques

\section{AUTEUR}

\section{CHRISTOPHE DEGUEURCE}

Professeur, conservateur du musée Fragonard, Université Paris-Est, École nationale vétérinaire d'Alfort cdegueurce@vet-alfort.fr 